\title{
Principales componentes del Trastorno Bipolar
}

\author{
Main components of Bipolar Disorder
}

\author{
Lizeth Hernández-Rosalio ${ }^{a}$
}

\begin{abstract}
:
Mental disorders such as Bipolar Disorder (TB) have increased their incidence in the last decade worldwide, so currently in Mexico; they represent a great challenge in the area of Psychiatry due to the high mortality rate due to suicides committed by the patients suffering from a Mental Disorder. This study is based on updated scientific review sources where it is intended to update and disclose the main genetic, chemical, and environmental risk factors that intervene etiologically in the production of mental instability that could subsequently trigger the presence of a Bipolar Disorder. , to publicize the clinical manifestations of this disease to provide an effective and timely diagnosis in patients, so that they return to their daily activities and not be disabling in their daily life.
\end{abstract}

Keywords:

Mania, hippomania, depression, suicide.

Resumen:

Los Trastornos mentales como lo es el Trastorno Bipolar (TB) han incrementado su incidencia en la última década a nivel mundial, por ello actualmente en México representan un gran reto en el área de Psiquiatría debido a la alta tasa de mortalidad por suicidios que cometen los pacientes que padecen algún Trastorno Mental. El presente estudio se basa en fuentes de revisión de carácter científico actualizado donde se pretende actualizar y divulgar los principales factores de riesgo genéticos, químicos, o medioambientales que intervienen de manera etiológica en la producción de inestabilidad mental que posteriormente podrían desencadenar la presencia de un Trastorno Bipolar, dar a conocer las manifestaciones clínicas de esta enfermedad para poder otorgar un diagnóstico eficaz y oportuno en los pacientes, con el fin de que regresen a sus actividades cotidianas y no le resulte incapacitante en su vida diaria.

\section{Palabras Clave:}

Manía, hipomanía, depresión, suicidio.

\section{Introducción}

El Trastorno Bipolar (TB) también denominado Espectro Bipolar o Trastorno Afectivo Bipolar es una enfermedad psiquiátrica grave, caracterizada por fluctuaciones del estado de ánimo de las personas que lo padecen alternando episodios depresivos con episodios de manía o hipomanía. Este trastorno se caracteriza por ser de inicio temprano, pues se presenta en la adultez joven entre los 20 y 22 años de edad, sin embargo diversos estudios estiman que al momento de ser diagnosticado han transcurrido al menos 10 años desde el primer episodio de origen de la enfermedad, puesto que desafortunadamente los pacientes suelen acudir al médico hasta que la enfermedad resulta incapacitante en su entorno; también se caracteriza por tener un curso crónico y recurrente, pues constantemente están teniendo estos cambios en el estado de ánimo y al igual que la mayoría de las patologías de origen mental no se cura, solo vamos a controlar al paciente para que tenga una calidad de vida óptima y pueda reintegrarse a sus actividades cotidianas. 1,2

\section{Epidemiología}

Actualmente se estima que la prevalencia del trastorno bipolar es de 2 a $2.4 \%$ a nivel mundial, estadísticas sumamente importantes puesto que las tasas de suicidio entre personas con trastorno bipolar son de 20 a 30 veces

Autor de correspondencia, Universidad Autónoma del Estado de Hidalgo, https://orcid.org/0000-0001-7770-1735, Email: he375425@uaeh.edu.mx 
más altas que las tasas en la población general. En México, las cifras son bastante desfavorables pues se estima que la prevalencia a padecer cualquier trastorno afectivo a lo largo de la vida alcanza hasta el $9.2 \%$; de los cuales aproximadamente un tercio de los pacientes inician la enfermedad en la segunda infancia, que va de los 6 a los 12 años y su diagnóstico se realiza al menos 10 años después teniendo una edad media de presentación a los 22 años de edad, el inicio temprano se ha relacionado con cursos graves de la enfermedad y peor pronóstico a largo plazo. Por otro lado, la proporción entre hombres y mujeres es de 1:1, sin embargo, los episodios depresivos, mixtos y de ciclación rápida son más frecuentes en mujeres, mientras que los episodios maníacos son más frecuentes en hombres. ${ }^{2,3,4}$

\section{Etiología y Origen de la Enfermedad}

A lo largo del tiempo este padecimiento se ha asociado a un carácter evolutivo, pues ninguna otra especie padece este trastorno, se estima que "es el precio" que el ser humano debe pagar por ser una especie especializada en la percepción y entendimiento de la realidad. La predisposición genética de los pacientes a este trastorno se ha visto tiene un patrón no mendeliano pues el riesgo a padecerlo incrementa aproximadamente del 60 al $80 \%$ en los pacientes con algún familiar que lo presente. ${ }^{5}$

La patología se puede entender como una serie de eventos concatenados, la cual provoca un desequilibrio en el organismo manifestándose con la irregularidad del ritmo sueño-vigilia, el cronotipo vespertino, irregularidades de las señales del tiempo social, alteraciones en el eje hipotálamo-pituitario-adrenal, y alteraciones en la emoción, sensación y percepción. ${ }^{6,7,8}$

Se han identificado al menos 30 loci asociados al trastorno bipolar que codifican canales iónicos, transportadores de neurotransmisores y componentes sinápticos; los genes más estudiados y con más relación son: ${ }^{9}$

CACNA1C: Codificador de la subunidad a1 de los canales de calcio tipo $L$, relacionado a la entrada de calcio e hiperpolarización celular.

ANK3: Codifica anquirina G, que acopla los canales de sodio axonales activados por voltaje al citoesqueleto y también tiene funciones en las dendritas y la glía.

ODZ4: Proteína transmembrana de teneurina 4 que durante el desarrollo del cerebro parece jugar un papel central en la regulación de la conectividad neuronal y sináptica y se relaciona con esquizofrenia, autismo, depresión y déficit de atención.

NEUROCAN (NCAN): Codifica una proteína que participa en la modulación de la adhesión celular, la migración celular y la guía de los axones y tiene relación con el TB.

\section{Cuadro Clínico}

Dentro de sus características clínicas los pacientes suelen debutar las diferentes fases de la enfermedad posterior a la exposición de eventos que hacen revolucionar su mente pasando de un estado depresivo a episodios de hipomanía o manía donde su humor se encuentra sumamente elevado con una marcada distorsión de la realidad, y puede variar desde una jovialidad despreocupada a una excitación casi incontrolable con euforia. En estos pacientes su autoestima se encuentra sumamente aumentada, con ideas de grandeza y exceso de confianza en sí mismo presentando incluso inhibiciones sociales normales dando lugar a comportamientos desvergonzados. ${ }^{10,11,12}$

Por el contrario, cuando estos pacientes cursan con cuadros de Depresión son caracterizados por tener un marcado decaimiento del estado de ánimo hasta el punto de incapacitarlos para levantarse de la cama, habitualmente el sueño se halla perturbado pues el paciente suele dormir por más de 15 horas y casi siempre decae su apetito y la confianza en sí mismo, muy a menudo aparecen algunas ideas de culpa, de ser inútil o de suicidio. ${ }^{13,14}$

El 3 de septiembre de 1999 en la Clínica Psiquiátrica de Norte América, Akiskal, propone una clasificación enfatizando el concepto de "Espectro" sobre la clínica, los antecedentes premórbidos y los temperamentos de las enfermedades del humor.

La revisión que hace Akiskal, ${ }^{15}$ sobre el espectro bipolar los clasifica como:

- Bipolar 1/2: trastorno esquizoafectivo tipo bipolar

- Bipolar I: al menos un episodio de manía o mixto

- Bipolar I 1/2: depresiones con hipomanías prolongadas

- Bipolar II: hipomanía y depresión

- Bipolar II 1/2: depresiones con inestabilidad anímica

- Bipolar III: depresiones con hipomanía inducida por fármacos

- Bipolar III 1/2: cambios anímicos por abuso de sustancias / alcohol

- Bipolar IV: depresión tardía en temperamento hipertímico

- Tipo V: depresiones cíclicas recurrentes (>5) con síntomas hipomaníacos, antecedentes familiares de bipolaridad y respuesta más a estabilizantes

- Tipo VI: inestabilidad anímica y/o hipomanías en pacientes con demencia temprana. 


\section{Diagnóstico}

Para realizar la valoración de un paciente se requiere la ayuda de un psiquiatra debido a las dificultades que entrañan el manejo del interrogatorio y todo el proceso diagnóstico. ${ }^{16,17}$ En un paciente con alta sospecha de Trastorno Bipolar las preguntas clave a realizar son: "¿alguna vez ha tenido un exceso marcado de energía?" de ser así; "¿es lo suficientemente alto para no querer dormir?". Dado que puede ser complejo interrogar directamente al paciente, se puede recabar información con los familiares o terceras personas. El médico debe cerciorarse que los síntomas no se deban a otros padecimientos como la intoxicación por drogas o lesión en lóbulo frontal tras algún traumatismo o presencia de masa neoplásica, otros diagnósticos diferenciales son la esquizofrenia o el síndrome de déficit de atención con hiperactividad. Actualmente existen métodos de ayuda para identificar los síntomas de manía, hipomanía o depresión en el paciente; a través de cuestionarios 0 autoevaluaciones como lo es la escala de manía de Young o la escala de Hamilton..$^{18,19}$

Dentro de los cambios neurobiológicos que podemos corroborar por imagen se incluyen: hipertrofia ventricular, especialmente del tercer ventrículo, atrofia cerebelar, prominencia de los surcos corticales, hiperintensidades subcorticales, reducción del volumen de la corteza frontal en la sustancia gris, hipocampal y de la amígdala.

La importancia de identificar los puntos clave de la patología es debido a que hasta $69 \%$ de las personas con TB reciben un diagnóstico inicial incorrecto y la demora en el diagnóstico adecuado puede alcanzar hasta 10 años o más. ${ }^{20,21,22}$

\section{Pronóstico y Tratamiento}

El carácter crónico y recurrente de este trastorno conlleva implícito un deterioro funcional y cognitivo al cual se le asocia una disminución en el tiempo y la calidad de vida pues existe un alto incremento en la mortalidad de estos pacientes, especialmente por suicidio y enfermedad cardiovascular. En el tratamiento del trastorno bipolar existen dos puntos de apoyo, el primero es la terapia farmacológica y la segundo son las intervenciones psicosociales que constituyen la base para el tratamiento exitoso, dentro de las ultimas se recomienda la psicoeducación a todos los pacientes y miembros de la familia para la prevención de recaídas. En lo que respecta al manejo médico, a ningún paciente se le debe evaluar solo, siempre debe acompañarlo un familiar y se le debe explicar al paciente y familiares la suspensión de cualquier tipo de alcohol o estimulantes como la cafeína, el tabaco o cualquier tipo de droga. ${ }^{23,24}$

Como tratamiento farmacológico se utilizan estabilizadores de la membrana neuronal como el valproato semisódico (valprosid R $500 \mathrm{mg}$ ), el topiramato, la lamotrigina, con mucha reserva se utiliza el litio que es un catión monovalente y es el mejor eutimizante conocido, este medicamento tiene una presentación en tabletas de $300 \mathrm{mg}$ y la dosis puede llegar hasta $1200 \mathrm{mg} / \mathrm{dí}^{2} .{ }^{25,26}$ La carbamazepina es un medicamento de segunda línea eficaz en la manía, aunque puede llegar a presentar efectos adversos de tolerabilidad y seguridad, puede inducir erupción cutánea, síndrome de Stevens-Johnson, necrólisis epidérmica. Otros medicamentos utilizados son los antipsicóticos atípicos, como la quetiapina, olanzapina, y risperidona. ${ }^{27}$

\section{Conclusión}

El Trastorno Bipolar es una patología más frecuente de lo que quisiéramos en nuestro país, desafortunadamente su diagnóstico se realiza muy tarde en los pacientes que lo padecen pues la población suele no acudir al médico hasta que la patología les resulta incapacitante en su vida cotidiana; aunado a eso el Trastorno Bipolar comparte cuadro clínico con otras patologías psiquiátricas dependiendo de la etapa clínica en la que se encuentre lo cual a veces conlleva a hacer un diagnóstico erróneo de primera instancia, por ejemplo; cuando el paciente está en un periodo depresivo, debe diferenciarse con depresión unipolar, cuando éste mismo padecimiento presenta características maníacas o hipomaniacas se debe buscar diagnósticos diferenciales como la depresión por duelo no resuelto, pues esta patología puede originar una depresión psicótica o modular a un Trastorno Bipolar. La conducta suicida y la consumación del suicidio son muy frecuentes en el Trastorno Bipolar, siendo la segunda patología con la que más está relacionado, así como las conductas que ponen en riesgo la vida del paciente en la fase maniaca se deben de seguir, vigilar u sobre todo educar al paciente y su red de apoyo para el apego del tratamiento.

\section{Referencias}

[1] Agosti V, Stewart JW. Hypomania with and without Dysphoria: comparison of comorbidity and clinical characteristics of respondents from a national community sample. J Affect Disord. 2008;108(1-2):177-82.

[2] Akiskal H, Benazzi F, Perugi G, Rihmer Z. Depresión "unipolar" agitada reconceptualizada como un estado mixto depresivo: implicaciones para la controversia antidepresivo-suicidio. J afectar el desorden. 2005; 85 (3): 245-58.

[3] Akiskal H, Pinto O. The evolving bipolar spectrum. Psychiatr Clin North Am. 1999;22(3):517-34.

[4] Baldessarini R, Tondo L, Vázquez GH. Tratamiento farmacológico del trastorno bipolar del adulto. Mol Psychiatry. 2019; 24 (2): 198217. 
[5] Burkhardt E, Pfennig A, Leopold K. Constelaciones de riesgo clínico para el desarrollo de trastornos bipolares. Medicina (Kaunas). 2021, 57 (8): 792.

[6] Cudney E, Frey BN, Streiner DL, Minuzzi L, Sassi RB. Biological rhythms are independently associated with quality of life in bipolar disorder. Int J Bipolar Disord [Internet]. 2016 Mar 16 [cited 2021 Sep 9]; 4:9. Available from: https://www.ncbi.nlm.nih.gov/pmc/articles/PMC4792817/

[7] Cruz Ramírez KV, Benavides Solarte MF, Gómez Restrepo C. Elementos traza y trastorno afectivo bipolar. Univ Médica [Internet] 2017;58(2). Available from: http://www.redalyc.org/articulo.oa?id=231053764006

[8] D’Onofrio S, Mahaffey S, Garcia-Rill E. Role of calcium channels in bipolar disorder. Curr Psychopharmacol [Internet]. 2017 [cited 2021 Sep 9];6(2):122-35. Available from: https://www.ncbi.nlm.nih.gov/pmc/articles/PMC5771645/

[9] Esparza RP. Tratamiento farmacológico de la depresión: actualidades y futuras direcciones [Internet]. Medigraphic.com. [citado el 10 de septiembre de 2021]. Disponible en: https://www.medigraphic.com/pdfs/facmed/un-2017/un175b.pdf

[10] Fabbri C. The role of genetics in bipolar disorder. En: Bipolar Disorder: From Neuroscience to Treatment. Cham: Springer International Publishing; 2020. p. 41-60. https://www.ncbi.nlm.nih.gov/pmc/articles/PMC3181866/

[11] García-Blanco AC, Sierra P, Livianos L. Nosología, epidemiología y etiopatogenia del trastorno bipolar: Últimas aproximaciones. Psiquiatr Biol. 2014;21(3):89-94.

[12] Huber RS, Yurgelun-Todd DA. Neural mechanisms underlying suicide behavior in youth with bipolar disorder. Bipolar Disord [Internet]. 2020 Mar [cited 2021 Aug 19];22(2):193-4. Available from: https://www.ncbi.nlm.nih.gov/pmc/articles/PMC8216322/

[13] Inteligencia Emocional en Pacientes con Trastorno Bipolar y Trastorno Límite de la Personalidad. UNIVERSIDAD DE MURCIA [Internet]. Digitum.um.es. [citado el 10 de septiembre de 2021]. Disponible https://digitum.um.es/digitum/bitstream/10201/53706/1/Jos\%c3\%a9 $\% 20 \mathrm{Mar} \%$ c3\%ada\%20Salmer\%c3\%b3n\%20AriasCamis\%c3\%b3n\%20Tesis\%20Doctoral.pdf

[14] Jiménez Arriero, M. A., Palomo ÁLvarez, T., \& Rodríguez Jiménez, R. (s. f.). Fundamentos de Psiquiatría. Editorial Médica Panamericana.

[15] Lorenzo-Luaces L, Amsterdam JD, Soeller I, DeRubeis RJ. Rapid versus non-rapid cycling bipolar II depression: response to venlafaxine and lithium and hypomanic risk. Acta Psychiatr Scand. 2016;133(6):459-69.

[16] Martínez Hernández $O$, Montalván Martínez $O$, Betancourt Izquierdo Y. Trastorno Bipolar. Consideraciones clínicas y epidemiológicas. Rev Méd Electrón [Internet]. 2019 Mar-Abr [citado: 02/07/21];41(2). Disponible en: http://www.revmedicaelectronica.sld.cu/index.php/rme/article/view/ $3157 / 42$

[17] McIntyre RS, Berk M, Brietzke E, Goldstein BI, López-Jaramillo C, Kessing LV, et al. Bipolar disorders. Lancet.
2020;396(10265):1841-56.

https://pubmed.ncbi.nlm.nih.gov/33278937/

[18] McCarty R, Josephs T, Kovtun O, Rosenthal SJ. Enlightened: addressing circadian and seasonal changes in photoperiod in animal models of bipolar disorder. Transl Psychiatry. 2021;11(1):373.

[19] Mullan KA, Anderson A, Illing PT, Kwan P, Purcell AW, Mifsud NA. HLA-associated antiepileptic drug-induced cutaneous adverse reactions. HLA. 2019;93(6):417-35.

[20] Ramírez Vargas, X., Arias Leal, M. L., \& Madrigal Zamora, S. (2020). Actualización del trastorno afectivo bipolar. Revista Médica Sinergia, 5(9), e572.

[21] Selle V, Schalkwijk S, Vázquez GH, Baldessarini RJ. Tratamientos para la depresión bipolar aguda: metanálisis de ensayos controlados con placebo en monoterapia de anticonvulsivos, litio y antipsicóticos. Farmacopsiquiatría. 2014; 47 (2): 43-52.

[22] Suliman M, Schmidtke MW, Greenberg ML. El papel de la vía UPR en la fisiopatología y el tratamiento del trastorno bipolar. Neurosci de células frontales. 2021; 15: 735622 .

[23] Scaini G, Valvassori SS, Diaz AP, Lima CN, Benevenuto D, Fries GR, et al. Neurobiology of bipolar disorders: a review of genetic components, signaling pathways, biochemical changes, and neuroimaging findings. Braz J Psychiatry [Internet]. 2020 Apr 3 [cited 2021 Aug 19];42(5):536-51. Available from: https://www.ncbi.nlm.nih.gov/pmc/articles/PMC7524405/

[24] Takaesu Y. Circadian rhythm in bipolar disorder: A review of the literature. Psychiatry Clin Neurosci. 2018 Sep;72(9):673-82.

[25] Vázquez GH, Holtzman JN, Tondo L, Baldessarini RJ. Eficacia y tolerabilidad de los tratamientos para la depresión bipolar. J afectar el desorden. 2015; 183: 258-62.

[26] Vázquez GH, Strejilevich S, García Bonetto G, Cetkovich-Bakmas M, Zaratiegui R, Lagomarsino A, et al. Argentine consensus on the treatment of bipolar disorders. Vertex. 2005;16 Suppl:3-24

[27] Yatham LN, Kennedy SH, Parikh SV, Schaffer A, Bond DJ, Frey BN, et al. Canadian Network for Mood and Anxiety Treatments (CANMAT) and International Society for Bipolar Disorders (ISBD) 2018 guidelines for the management of patients with bipolar disorder. Bipolar Disord. 2018;20(2):97-170. 